\title{
Game Perspectives of DEA Models and Their Duals
}

\author{
Fang Xu, ${ }^{1}$ DaQun Zhang, ${ }^{2}$ GuoLiang Yang, ${ }^{1}$ and WenBin Liu ${ }^{3}$ \\ ${ }^{1}$ Institute of Policy and Management, Chinese Academy of Sciences, Beijing 100080, China \\ ${ }^{2}$ Department of Accounting, Temple University, Philadelphia, PA 19122, USA \\ ${ }^{3}$ Kent Business School, University of Kent, Canterbury CT 7PE, UK \\ Correspondence should be addressed to WenBin Liu; w.b.liu@kent.ac.uk
}

Received 22 September 2013; Accepted 7 November 2013

Academic Editor: Pei-ai Zhang

Copyright (C) 2013 Fang Xu et al. This is an open access article distributed under the Creative Commons Attribution License, which permits unrestricted use, distribution, and reproduction in any medium, provided the original work is properly cited.

\begin{abstract}
We present a series of two-person games, which lead to various DEA models. The relationship between the DEA models and the games is explicit in our setting, although the Nash equilibrium solutions do not generally exist. Besides the classic DEA models, we also establish an explicit relationship between the games and some extended DEA models, such as free disposable hull DEA models and "negative" or "inverted" DEA models.
\end{abstract}

\section{Introduction}

DEA has widely been used in performance evaluation or productivity evaluation. The DEA models have been derived from different perspectives like econometrics and operations research; see for example, Charnes et al. [1], Banker et al. [2], and Liu et al. [3] for the details. Also, several researchers found that there are close connections between DEA and the game theory [4]. As a pioneer, Banker et al. [5, 6] explored the connections between DEA and the game theory. In their work, they first built the following payoff matrix:

$$
e_{i j}=\frac{y_{0} / x_{i 0}}{y_{j} / x_{i j}} .
$$

In their formulation, the $\mathrm{DMU}_{0}$ being evaluated acts as player 1, who wishes to maximize its payoff and has pure strategies defined by the selection of some inputs $\{i\}$. On the other hand, player 2 acts as a "central evaluator," who wishes to minimize the payoff of player 1 and has pure strategies defined by the selection of a competitive DMU $\{j\}$. Then, the authors used a mixed strategy formed from the pure strategies and computed an expected payoff function constructed from the finite payoff matrix above. They formulated the game model as the pair of linear programming problems shown in Table 1.

After several transformations of the problem $(*)$, the authors obtained the "CCR ratio form," which is also the maximal expected gains that $\mathrm{DMU}_{0}$ can obtain from the corresponding payoff matrix. However, the game model developed by Banker [5] requires that the output in the model is a number. Banker et al. [6] extended their initial work to include multiple outputs, but they had to replace the original CCR model with the BCC model, which makes it possible to write the linear programming problem for player 2 directly.

To establish a rigorous connection between the CCR model and the game theory, Rousseau and Semple [7] developed a class of two-person ratio efficiency games, which have ratio payoff functions solved by two equivalent primal and dual linear programming problems. The authors argued that "the richest information is available from the primaldual linear programs: one side emphasizes "envelopment" multipliers, the other emphasizes input-output multipliers." And the most general form of the games is described in Table 2.

The $\mathrm{DMU}_{0}$, the unit being evaluated, acts as player 1 . And the intention of player 1 is to choose some optimal weights $\left(u^{*}, v^{*}\right)$ in $P$ and minimize the fractional payoff of player 2. Meanwhile, Player 2 acts as a regulator who will select some $\lambda$ in $Q$ to construct a virtual "aggregate competitor" and maximize its worst case payoff under all the possible weights chosen by player 2 . The authors proved that $\left(u^{*}, v^{*}, \lambda^{*}\right)$ constitutes a saddle point for the ratio payoff function in (\#) and (\#\#). However, the games (\#) and (\#\#) cannot derive CCR model directly and only can yield 
TABLE 1

\begin{tabular}{|c|c|c|c|}
\hline Player 1 & & Player 2 & \\
\hline $\begin{array}{lc}\text { Max } & \rho_{0} \\
\text { subject to }- & \sum_{i=1}^{m} p_{i} e_{i j}+\rho_{0} \leq 0 \\
& \sum_{i=1}^{m} p_{i}=1, p_{i} \geq 0 .\end{array}$ & $(*)$ & $\begin{array}{l}\text { Min } \quad \eta_{0} \\
\text { subject to }-\sum_{j=1}^{n} q_{j} e_{i j}+\eta_{0} \geq 0 \\
\sum_{j=1}^{n} q_{j}=1, q_{j} \geq 0 .\end{array}$ & $(* *)$ \\
\hline
\end{tabular}

TABLE 2

\begin{tabular}{|c|c|c|c|c|c|}
\hline \multicolumn{3}{|l|}{ Player 1} & \multicolumn{3}{|c|}{ Player 2} \\
\hline$\underset{(u, v) \in P}{\operatorname{Min}} \max _{\lambda \in Q}$ & $\frac{\left(\sum_{j=1}^{n} \lambda_{j} Y_{j}\right)^{T} v}{\left(\sum_{j=1}^{n} \lambda_{j} X_{j}\right)^{T} u}$ & $(\#)$ & $\operatorname{Max}_{\lambda \in Q} \min _{(u, v) \in P}$ & $\left\{\frac{\left(\sum_{j=1}^{n} \lambda_{j} Y_{j}\right)^{T} v}{\left(\sum_{j=1}^{n} \lambda_{j} X_{j}\right)^{T} u}\right.$ & (\#\#) \\
\hline
\end{tabular}

Where $P=\left\{(u, v): u \geq 0, v \geq 0, v^{T} Y_{0} / u^{T} X_{0}=1\right\}$ and $Q=\left\{\lambda: \sum_{j=1}^{n} \lambda_{j}=1, \lambda_{j} \geq 0\right\}$ are two infinite pure strategy spaces.

the information necessary to conduct the CCR efficiency analysis.

To incorporate value judgment, Semple [8] extended the "two-person ratio efficiency game" by including polyhedral cone constraints in the pure strategies played by each player. And the connection between the game theory and DEA has been extended to include the cone ratio models (see $[9,10])$.

In another way, Hao et al. [11] followed the line of Banker's work, which needs to use the payoff matrices and mixed strategies, but extended the two-person zero-sum game to the closed convex cone constraints. Then, the authors proved the connection between the proposed convex cone constrained game model and the generalized DEA model.

In this paper, we will present a game perspective of DEA model using a different payoff function. We will show that many DEA models, including the CCR, BCC, and other models, can be derived from a series of min-max problems. We also show that some max-min problems will derive new DEA models.

Similarly, we assume that there are two players in our game. One player is the $\mathrm{DMU}_{0}$ being evaluated and the other player is a central evaluator. In this game, $\mathrm{DMU}_{0}$ wishes to maximize (or minimize) its gain (or loss). On the contrary, player 2 wants to minimize (or maximize) its loss (or gain). Corresponding to the players, there are two pure strategy spaces in the game. One is the weight space $W$ in the multiplier formulation of DEA and the other one is the index $\{j\}$, which selects a competitive $\mathrm{DMU}_{j}$. Clearly, such two strategy spaces are not "symmetric" in the sense that the second is smaller and thus has a weaker influence on the game results. In order to have a fairer game, one remedy is to enlarge it. For example, one can expand it into selections of all virtual DMUs. That is precisely the strategy space in Semple [8]. However, the connections between DEA models and the min-max or max-min programming become less clear and more complicated. In the following, we explain another idea to handle this issue.

For any game, $\max _{y \in Y} \min _{x \in X} \phi(x, y) \leq \min _{x \in X} \max _{y \in Y}$ $\phi(x, y)$ always holds, where $\phi(x, y)$ is the loss function of player 1 and $X, Y$ are the pure strategy spaces of player 1 and player 2, respectively. When $\max _{y \in Y} \min _{x \in X} \phi(x, y)=$ $\min _{x \in X} \max _{y \in Y} \phi(x, y)$, we say that in this game there exists at least one Nash equilibrium. The existence of the Nash equilibriums shows the min-max/max-min that is an optimal strategy for both players, and the game may have no solution if the Nash equilibriums do not exist. However, the min-max strategy is the safest strategy for player 1 if player 2 has the right to terminate the game (so it has the right to select the strategies for the final step of the game), and the max-min strategy is the safest strategy for player 2 if player 1 has the right. In our case, Nash equilibriums generally do not exist since the second strategy space is too small. We compensate this by assuming that the player whose strategy space is the second has the right to terminate the game. Thus, in the present situation, the min-max strategies should be the safest for player 1. The advantage of this approach seems is that many different DEA models can be directly connected to the safest strategies of the player.

The paper is organized as follows. To illustrate the main ideas clearly, Section 2 presents the min-max and max-min formulations for DEA models with index data at first. In Section 3, we discuss the general case of the min-max and max-min formulations for the CCR, BCC, and other DEA models, and the conclusions and discussions are given in Section 4 .

\section{A Game Perspective of Index DEA Models}

We start our investigation from a class of simpler DEA models, where the inputs are assumed to be standardized, or DEA models without inputs. This class of DEA models in fact uses index data and therefore is referred to as index DEA models; see Lovell and Pastor [12], Halkos and Salamouris [13], and Liu et al. [3] for more details.

2.1. Min-Max Problems of Index DEA Models. In this paper, a pure strategy of weights is denoted by a vector $\mathbf{u}=$ $\left(u_{1}, \ldots, u_{s}\right)^{t}$ in a weight space $W$. Now, let us consider the following case: the pure strategy of $\mathrm{DMU}_{0}$ is the weight space. This means that $\mathrm{DMU}_{0}$ can freely select weights from the weight space to account its weighed output score $\sum_{r=1}^{s} u_{r} y_{r 0}$. Due to the conflicting interests, the evaluator may not be happy to use these weights for accounting their scores. Here, we assume that they can select a $\mathrm{DMU}_{j}$ from competitive DMUs' space $J=\{1, \ldots, n\}$ to increase the relative loss function of $\sum_{r=1}^{s} u_{r} y_{r j} / \sum_{r=1}^{s} u_{r} y_{r 0}$ to force $\mathrm{DMU}_{0}$ to change 
its strategies. Obviously, the larger is $\sum_{r=1}^{s} u_{r} y_{r j} / \sum_{r=1}^{s} u_{r} y_{r 0}$, the worse is the relative score of $\mathrm{DMU}_{0}$.

If the $\mathrm{DMU}_{0}$ is risk aversion, then the $\mathrm{DMU}_{0}$ will consider the possible worst case; that is, player 2 will choose best practice $\mathrm{DMU}_{j}$ to maximize the performance gap with $\mathrm{DMU}_{0}$. Then the $\mathrm{DMU}_{0}$ will choose a weight that will minimize the gap with all the possible competitive $\mathrm{DMU}_{j}$. Under this circumstance, $\mathrm{DMU}_{0}$ will choose the "min-max" strategy. In the following mathematical formulation, we will use the normalization $\left\{u \mid \sum_{r=1}^{s} u_{r} y_{r 0}=1, u_{r} \geq 0\right\}$, thus, $\sum_{r=1}^{s} u_{r} y_{r j} / \sum_{r=1}^{s} u_{r} y_{r 0}$ can be simplified as $\sum_{r=1}^{s} u_{r} y_{r j}$, and then the min-max strategy illustrated above can be written as follows:

$$
\min _{u \in W} \max _{j \in J} \sum_{r=1}^{s} u_{r} y_{r j}
$$

where $W=\left\{u \mid \sum_{r=1}^{s} u_{r} y_{r 0}=1, u_{r} \geq 0\right\}$ and $J=\{1, \ldots, n\}$.

For the purpose of analysis, we introduce an auxiliary variable $v$ such that $v=\operatorname{Max}_{j=1, \ldots, n}\left\{\sum_{r=1}^{\mathcal{s}} u_{r} y_{r j}\right\}$. Then, model (2) may be rewritten as

$$
\begin{array}{ll}
\underset{u}{\operatorname{Min}} & v \\
\text { subject to } & \sum_{r=1}^{s} u_{r} y_{r j} \leq v, \\
& \sum_{r=1}^{s} u_{r} y_{r 0}=1, \\
& u_{r} \geq 0, \quad j=1, \ldots, n .
\end{array}
$$

And the optimal value $v^{*}$ is the worst possible relative score of $\mathrm{DMU}_{0}$. Now, we consider its dual model, which will be shown to be the following index DEA model:

$$
\begin{array}{ll}
\operatorname{Max} & \theta \\
\text { subject to } & \sum_{j=1}^{n} Y_{j} \lambda_{j} \geq \theta y_{0}, \\
& \sum_{j=1}^{n} \lambda_{j}=1, \\
& \lambda_{j} \geq 0, \quad j=1, \ldots, n .
\end{array}
$$

Model (4) is first introduced by Lovell and Pastor [12].

Proposition 1. Model (4) is the dual model of model (3).

Proof. Model (3) can be rewritten as follows:

$$
\begin{array}{ll}
\text { Min } & v \\
\text { subject to } & Y^{T} u-v I \leq 0 \\
& -Y_{0}^{T} u+1=0 \\
& u \geq 0
\end{array}
$$

where $Y_{0}=\left(y_{10}, \ldots, y_{s 0}\right)^{T}, Y=\left(Y_{1}, \ldots, Y_{n}\right), \mathbf{u}=$ $\left(u_{1}, \ldots, u_{s}\right)^{T}$, and $I=(1, \ldots, 1)^{T}$. The Lagrange function of model (5) is $L(u, v, \theta, \lambda)=v+\lambda^{T}\left(Y^{T} u-v I\right)+\theta\left(-Y_{0}^{T} u+1\right)$, and its dual function is

$$
\begin{aligned}
q(\theta, \lambda) & =\inf _{\mu \geq 0, v}\{L(\mu, \lambda)\} \\
& =\inf _{\mu \geq 0, v}\left\{\theta+\left(\lambda^{T} Y^{T}-\theta Y_{0}^{T}\right) u+\left(-\lambda^{T} I+1\right) v\right\} \\
& = \begin{cases}\theta, & \text { if }-\theta Y_{0}^{T}+\lambda^{T} Y^{T} \geq 0,-\lambda^{T} I+1=0, \\
-\infty, & \text { if }-\theta Y_{0}^{T}+\lambda^{T} Y^{T} \leq 0,\left(-\lambda^{T} I+1\right) \neq 0 .\end{cases}
\end{aligned}
$$

Thus, the dual problem is

$$
\begin{array}{ll}
\text { Max } & q(\theta, \lambda) \\
\text { Subject to } & \lambda \geq 0,
\end{array}
$$

which reads

$$
\begin{array}{ll}
\operatorname{Max} & \theta \\
\text { subject to } & \sum_{j=1}^{n} Y_{j} \lambda_{j} \geq \theta Y_{0}, \\
& \sum_{j=1}^{n} \lambda_{j}=1, \\
& \lambda_{j} \geq 0, \quad j=1, \ldots, n .
\end{array}
$$

Hence, model (4) is the dual of model (3).

Let us investigate model (3) further. Let $\mu_{r}=u_{r} / v$, and then model (3) can be easily changed to the following model:

$$
\begin{array}{ll}
\operatorname{Max} & \sum_{r=1}^{s} \mu_{r} y_{r 0} \\
\text { subject to } & \sum_{r=1}^{s} \mu_{r} y_{r j} \leq 1, \\
& \mu_{r} \geq 0, \quad j=1, \ldots, n .
\end{array}
$$

Now, we can understand it by intuition: model (9) (therefore (4)) is simply an index DEA model, which selects weights to maximize its weighted score like a classical DEA model. Thus, the min-max strategy just leads to index DEA models.

2.2. Max-Min Problem of Index DEA. As discussed before, the min-max strategy leads to index DEA models. Now, we consider the "max-min" strategy as follows:

$$
\underset{j \in J}{\operatorname{Max}} \operatorname{Min}_{u \in W} \sum_{r=1}^{s} u_{r} y_{r j},
$$

where $W=\left\{u \mid \sum_{r=1}^{s} u_{r} y_{r 0}=1, u_{r} \geq 0\right\}$ and $J=\{1, \ldots, n\}$. We can calculate this model through the following two-stage algorithm.

First Stage. We can divide model (10) into $n$ separate models as follows:

$$
\begin{aligned}
h_{j}^{*}=\operatorname{Min}_{u} & \sum_{r=1}^{s} u_{r} y_{r j} \\
\text { subject to } & \sum_{r=1}^{s} u_{r} y_{r 0}=1, \\
& u_{r} \geq 0 .
\end{aligned}
$$


Let $h_{j}^{*}$ be the optimal value of (11). Then, we obtain all the infimum $\left\{h_{1}^{*}, h_{2}^{*}, \ldots, h_{n}^{*}\right\}$.

Second Stage. Let $h^{*}=\max _{j=1, \ldots, n}\left\{h_{j}^{*}\right\}$.

From the min-max theory, it always holds that $\operatorname{Max}_{j=1, \ldots, n} \operatorname{Min}_{u} \sum_{r=1}^{s} u_{r} y_{r j} \leq \operatorname{Min}_{u} \operatorname{Max}_{j=1, \ldots, n} \sum_{r=1}^{s} u_{r} y_{r j}$, where $\sum_{r=1}^{s} u_{r} y_{r 0}=1$ and $u \geq 0$. And if $\operatorname{Max}_{j=1, \ldots, n} \operatorname{Min}_{u} \sum_{r=1}^{s} u_{r} y_{r j}=\operatorname{Min}_{u} \operatorname{Max}_{j=1, \ldots, n} \sum_{r=1}^{s} u_{r} y_{r j}$, we can conclude that this game has a Nash equilibrium solution. Unfortunately, Nash equilibrium solution does not always exist actually unless $\mathrm{DMU}_{0}$ only has one reference peer in model (4). Let us consider the dual model of (11), which can be written as

$$
\begin{array}{ll}
\operatorname{Max} & \theta_{j} \\
\text { subject to } & Y_{j} \geq \theta_{j} Y_{0}
\end{array} .
$$

And considering $\theta^{*}=\max _{j=1, \ldots, n}\left\{\theta_{j}^{*}\right\}$, we can write its dual as

$$
\begin{array}{ll}
\operatorname{Max} & \theta \\
\text { subject to } & \sum_{j=1}^{n} Y_{j} \lambda_{j} \geq \theta Y_{0}, \\
& \sum_{j=1}^{n} \lambda_{j}=1, \\
& \lambda_{j}=1 \text { or } 0, \quad j=1, \ldots, n,
\end{array}
$$

which is a free disposable hull DEA model without inputs. Thus, in general, there is no Nash equilibrium solution for this game formulation. If the $\mathrm{DMU}_{0}$ had the right to terminate the game, assumed to be risk aversion, the central evaluator would adopt the max-min strategy in our situation, which derives a FDH DEA model.

2.3. Max-Min Problem of a Different Game. Now, we consider a different game, where $\mathrm{DMU}_{0}$ and the central evaluator exchange their pure strategy spaces. That is $\mathrm{DMU}_{0}$ can control the variable $j$, and the evaluator can control the weight $u$. This implies that $\mathrm{DMU}_{0}$ itself can select the worst practice $\mathrm{DMU}_{j}$ as its benchmark freely. On the contrary, the central evaluator would choose the best weights. As explained in Section 1, the safest strategy for the evaluator is the maxmin strategy, which can be modeled as follows:

$$
\underset{u \in W}{\operatorname{Max}} \operatorname{Min}_{j \in J} \sum_{r=1}^{s} u_{r} y_{r j},
$$

where $W=\left\{u \mid \sum_{r=1}^{s} u_{r} y_{r 0}=1, u_{r} \geq 0\right\}$ and $J=$ $\{1, \ldots, n\}$. By introducing an auxiliary variable $v=$ $\operatorname{Min}_{j=1, \ldots, n}\left\{\sum_{r=1}^{s} u_{r} y_{r j}\right\}$, the model above will be transformed to

$$
\begin{array}{ll}
\operatorname{Max}_{u_{r}} & v \\
\text { subject to } & \sum_{r=1}^{s} u_{r} y_{r j} \geq v, \\
& \sum_{r=1}^{s} u_{r} y_{r 0}=1, \\
& u_{r} \geq 0, \quad j=1, \ldots, n .
\end{array}
$$

And its dual model is as follows:

$$
\begin{array}{ll}
\text { Min } & \theta \\
\text { subject to } & \sum_{j=1}^{n} Y_{j} \lambda_{j} \leq \theta Y_{0}, \\
& \sum_{j=1}^{n} \lambda_{j}=1, \\
& \lambda_{j} \geq 0, \quad j=1, \ldots, n .
\end{array}
$$

Then, its meaning is clearer. Model (16) compares $\mathrm{DMU}_{0}$ with the worst DMU, instead of comparing it with the best one as in the classic DEA. This idea was first used by Takamura and Tone [14] and Yamada et al. [15]. Using transformations $\mu_{r}=$ $u_{r} / v$, then model (15) is changed to

$$
\begin{array}{ll}
\operatorname{Min}_{u_{r}} & \sum_{r=1}^{s} \mu_{r} y_{r 0} \\
\text { subject to } & \sum_{r=1}^{s} \mu_{r} y_{r j}=1, \\
& u_{r} \geq 0, \quad j=1, \ldots, n .
\end{array}
$$

Then, this idea is more clearly illustrated. For the purpose of relocating several government agencies out of Tokyo, Takamura and Tone [14] used both models (9) and (17) with weight restrictions to deal with the problem of site selection for that project. They argued that "Each site is compared with these worst performers and is gauged by its efficiency "negatives" as the ratio of distances from the "worst" frontiers in the same way as in ordinary DEA." Yamada et al. [15] named this worst side approach "Inverted DEA."

Similarly, the min-max strategy will lead to a free-disposal hull inverted DEA model.

\section{Min-Max/Max-Min Problem of DEA Models}

3.1. Min-Max/Max-Min Problem of CCR. After exploring the game perspective of DEA models with index data, we turn to the classical input-output DEA formulation. We first consider the same game situation set in Section 2.1 and use a similar relative loss function as before. Then, the min-max problem reads

$$
\underset{u, v \in W}{\operatorname{Min}} \operatorname{Max}_{j \in J} \frac{\sum_{r=1}^{s} u_{r} y_{r j} / \sum_{i=1}^{m} v_{i} x_{i j}}{\sum_{r=1}^{s} u_{r} y_{r 0} / \sum_{i=1}^{m} v_{i} x_{i 0}},
$$

where $W=\left\{u \mid \sum_{r=1}^{s} u_{r} y_{r 0}=1, u \geq 0\right\} \cup\{v \geq 0\}$ and $J=$ $\{1, \ldots, n\}$.

Let $t=\max _{j}\left\{\sum_{r=1}^{s} u_{r} y_{r j} / \sum_{r=1}^{s} v_{i} x_{i j}\right\}$. Then, we can transform the model above to the following model:

$$
\begin{array}{ll}
\operatorname{Min}_{u, v} & t \sum_{i=1}^{m} v_{i} x_{i 0} \\
\text { subject to } & \frac{\sum_{r=1}^{s} u_{r} y_{r j}}{\sum_{i=1}^{m} v_{i} x_{i j}} \leq t, \\
& \sum_{r=1}^{s} u_{r} y_{r 0}=1, \\
& u \geq 0, \quad v \geq 0, \quad j=1, \ldots, n .
\end{array}
$$


Further, let $v_{i}=v_{i} t$, and then the model is further transformed to

$$
\begin{array}{ll}
\underset{u, v}{\operatorname{Min}} & \sum_{i=1}^{m} v_{i} x_{i 0} \\
\text { subject to } & \sum_{r=1}^{s} u_{r} y_{r j}-\sum_{i=1}^{m} v_{i} x_{i j} \leq 0, \\
& \sum_{r=1}^{s} u_{r} y_{r 0}=1, \\
& u \geq 0, \quad v \geq 0, \quad j=1, \ldots, n .
\end{array}
$$

This is the standard output-oriented CCR DEA model. If we replace the normalization $\sum_{r=1}^{s} u_{r} y_{r 0}=1$ with $\sum_{i=1}^{m} v_{i} x_{i 0}=$ 1 , then after several similar transformations as above, we will derive the input-oriented CCR model. follows:

Similarly, we can examine the max-min strategy as

$$
\operatorname{Max}_{j \in J} \operatorname{Min}_{u, v \in W} \frac{\sum_{r=1}^{s} u_{r} y_{r j} / \sum_{i=1}^{m} v_{i} x_{i j}}{\sum_{r=1}^{s} u_{r} y_{r 0} / \sum_{i=1}^{m} v_{i} x_{i 0}}
$$

where $W=\left\{u \mid \sum_{r=1}^{s} u_{r} y_{r 0}=1, u \geq 0\right\} \cup\{v \geq 0\}$ and $J=\{1, \ldots, n\}$. We again calculate the model through the following two-stage algorithm.

First Stage. We divide model (21) into $n$ separate models as follows:

$$
\begin{aligned}
h_{j}^{*}=\operatorname{Min}_{u, v} & \sum_{i=1}^{m} v_{i} x_{i 0} \\
\text { subject to } & \sum_{r=1}^{s} u_{r} y_{r j}-\sum_{i=1}^{m} v_{i} x_{i j} \leq 0, \\
& \sum_{r=1}^{s} u_{r} y_{r 0}=1, \\
& u \geq 0, \quad v \geq 0 .
\end{aligned}
$$

And let $h_{j}^{*}$ be the optimal value of model (22). Then, we obtain all the infimum $\left\{h_{1}^{*}, h_{2}^{*}, \ldots, h_{n}^{*}\right\}$.

Second Stage. Let $h^{*}=\max _{j=1, \ldots, n}\left\{h_{j}^{*}\right\}$.

Then, we consider the dual model of (22) as before, which can be written as

$$
\begin{array}{ll}
\operatorname{Max} & \theta_{j} \\
\text { subject to } & X_{j} \lambda_{j} \leq X_{0}, \\
& Y_{j} \lambda_{j} \geq \theta_{j} Y_{0}, \\
& \lambda_{j} \geq 0 .
\end{array}
$$

And we know $\theta^{*}=\max _{j=1, \ldots, n}\left\{\theta_{j}^{*}\right\}$. Then, the programming problem above can be transformed further to

$$
\begin{array}{ll}
\text { Max } & \theta \\
\text { subject to } & \sum_{j=1}^{n} X_{j} \lambda_{j} \leq X_{0}, \\
& \sum_{j=1}^{n} Y_{j} \lambda_{j} \geq \theta Y_{0}, \\
& \lambda_{j}=0 \quad \text { or any other positive real number. } \\
& \text { And for } j=1, \ldots, n, \text { there is only one } \lambda_{j} \text { not zero. }
\end{array}
$$

This can be viewed as the FDH CCR DEA model.
3.2. Min-Max/Max-Min Problem of BCC. To derive the minmax/max-min problem of BCC model, we can change the relative loss function as follows:

$$
\begin{array}{ll}
\operatorname{Min}_{u_{,}, u_{0}} \operatorname{Max}_{j=1, \ldots, n} & \frac{\sum_{r=1}^{s} u_{r} y_{r j} /\left(\sum_{i=1}^{m} v_{i} x_{i j}+u_{0}\right)}{\sum_{r=1}^{s} u_{r} y_{r 0} /\left(\sum_{i=1}^{m} v_{i} x_{i 0}+u_{0}\right)} \\
\text { subject to } & \sum_{r=1}^{s} u_{r} y_{r 0}=1, \\
& u \geq 0, \quad v \geq 0, \quad u_{0} \text { is free. }
\end{array}
$$

Again let $t=\max _{j}\left\{\sum_{r=1}^{s} u_{r} y_{r j} /\left(\sum_{i=1}^{m} v_{i} x_{i j}+u_{0}\right)\right\}$. Then, we transform the model above to the following:

$$
\begin{array}{ll}
\operatorname{Min}_{u, u_{0}} & t\left(\sum_{i=1}^{m} v_{i} x_{i 0}+u_{0}\right) \\
\text { subject to } & \frac{\sum_{r=1}^{s} u_{r} y_{r j}}{\left(\sum_{i=1}^{m} v_{i} x_{i j}+u_{0}\right)} \leq t, \\
& \sum_{r=1}^{s} u_{r} y_{r 0}=1, \quad j=1, \ldots, n, \\
& u \geq 0, \quad v \geq 0, \quad u_{0} \text { is free. }
\end{array}
$$

Then, further let $v_{i}=t v_{i}$ and $u_{0}=t u_{0}$. Then, the model is transformed to

$$
\begin{array}{ll}
\operatorname{Min}_{u, v} & \sum_{i=1}^{m} v_{i} x_{i 0}+u_{0} \\
\text { subject to } & \sum_{r=1}^{s} u_{r} y_{r j}-\sum_{i=1}^{m} v_{i} x_{i j}-u_{0} \leq 0, \\
& \sum_{r=1}^{s} u_{r} y_{r 0}=1, \quad j=1, \ldots, n, \\
& u \geq 0, \quad v \geq 0, \quad u_{0} \text { is free. }
\end{array}
$$

This is the standard output-oriented BCC DEA model.

Similarly the "max-min" strategy leads to the following formulation:

$$
\begin{aligned}
\operatorname{Max}_{j=1, \ldots, n} \operatorname{Min}_{u_{1}, u_{0}} & \frac{\sum_{r=1}^{s} u_{r} y_{r j} /\left(\sum_{i=1}^{m} v_{i} x_{i j}+u_{0}\right)}{\sum_{r=1}^{s} u_{r} y_{r 0} /\left(\sum_{i=1}^{m} v_{i} x_{i 0}+u_{0}\right)} \\
\text { Subject to }: & \sum_{r=1}^{s} u_{r} y_{r 0}=1, \\
& u \geq 0, \quad v \geq 0, \quad u_{0} \text { is free. }
\end{aligned}
$$

Again, we can show that its dual reads

$$
\begin{array}{ll}
\operatorname{Max} & \theta \\
\text { subject to } & \sum_{j=1}^{n} X_{j} \lambda_{j} \leq X_{0}, \\
& \sum_{j=1}^{n} Y_{j} \lambda_{j} \geq \theta Y_{0}, \\
& \sum_{j=1}^{n} \lambda_{j}=1, \quad \lambda_{j}=1 \text { or } 0 .
\end{array}
$$


3.3. Max-Min Problem When Exchanging Strategy Spaces. Similar to Section 2.3, we exchange the pure strategies of $\mathrm{DMU}_{0}$ and central evaluator, and then the max-min formulation can be written as follows:

$$
\begin{aligned}
\underset{u, v}{\operatorname{Max}} \operatorname{Min}_{j=1, \ldots, n} & \frac{\sum_{r=1}^{s} u_{r} y_{r j} / \sum_{i=1}^{m} v_{i} x_{i j}}{\sum_{r=1}^{s} u_{r} y_{r 0} / \sum_{r=1}^{m} v_{i} x_{i 0}} \\
\text { subject to } & \sum_{i=1}^{m} v_{i} x_{i 0}=1, \\
& u \geq 0, \quad v \geq 0 .
\end{aligned}
$$

Letting $t=\min _{j}\left\{\sum_{r=1}^{s} u_{r} y_{r j} / \sum_{i=1}^{m} v_{i} x_{i j}\right\}$, we transform the model above to the following model:

$$
\begin{array}{ll}
\operatorname{Max}_{u, v} & \frac{t}{\sum_{r=1}^{s} u_{r} y_{r 0}} \\
\text { subject to } & \frac{\sum_{r=1}^{s} u_{r} y_{r j}}{\sum_{i=1}^{m} v_{i} x_{i j}} \geq t, \\
& \sum_{i=1}^{m} v_{i} x_{i 0}=1, \quad j=1, \ldots, n, \\
& u \geq 0, \quad v \geq 0 .
\end{array}
$$

Further, let $u_{r}=u_{r} / t$, and then we have

$$
\begin{array}{ll}
\operatorname{Max}_{u, v} & \frac{1}{\sum_{r=1}^{s} u_{r} y_{r 0}} \\
\text { subject to } & \sum_{r=1}^{s} u_{r} y_{r j}-\sum_{i=1}^{m} v_{i} x_{i j} \geq 0, \\
& \sum_{i=1}^{m} v_{i} x_{i 0}=1, \quad j=1, \ldots, n, \\
& u \geq 0, \quad v \geq 0 .
\end{array}
$$

$\mathrm{Or}$

$$
\begin{array}{ll}
\operatorname{Min}_{u, v} & \sum_{r=1}^{s} u_{r} y_{r 0} \\
\text { subject to } & \sum_{r=1}^{s} u_{r} y_{r j}-\sum_{i=1}^{m} v_{i} x_{i j} \geq 0, \\
& \sum_{i=1}^{m} v_{i} x_{i 0}=1, \quad j=1, \ldots, n, \\
& u \geq 0, \quad v \geq 0 .
\end{array}
$$

Proposition 2. The dual form of model (33) is as follows:

$$
\begin{array}{ll}
\operatorname{Max} & \theta \\
\text { subject to } & \sum_{j=1}^{n} x_{i j} \lambda_{j} \geq \theta x_{i 0}, \\
& \sum_{j=1}^{n} y_{r j} \lambda_{j} \leq y_{r 0}, \\
& \lambda_{j} \geq 0, \quad j=1, \ldots, n .
\end{array}
$$

Proof. Model (33) can be written as follows:

$$
\begin{array}{ll}
\text { Min } & Y_{0}^{T} u \\
\text { subject to } & Y^{T} u-X^{T} v \geq 0, \\
& -X_{0}^{T} v+1=0, \\
& u \geq 0, \quad v \geq 0,
\end{array}
$$

where $Y_{0}=\left(y_{10}, \ldots, y_{s 0}\right)^{T}, X_{0}=\left(x_{10}, \ldots, x_{m 0}\right)^{T}, \mathbf{Y}=$ $\left(Y_{1}, \ldots, Y_{n}\right), \mathbf{X}=\left(X_{1}, \ldots, X_{n}\right), \mathbf{u}=\left(u_{1}, \ldots, u_{s}\right)^{T}$, and $\mathbf{v}=\left(v_{1}, \ldots, v_{m}\right)^{T}$. The Lagrange function of model (35) reads: $L(u, v, \theta, \lambda)=Y_{0}^{T} u+\lambda^{T}\left(-Y^{T} u+X^{T} v\right)+\theta\left(-X_{0}^{T} v+1\right)$, and its dual function is

$$
\begin{aligned}
& q(\theta, \lambda) \\
& \quad=\inf _{u, v \geq 0}\{L(u, v, \theta, \lambda)\} \\
& \quad=\inf _{u, v \geq 0}\left\{\theta+\left(Y_{0}^{T}-\lambda^{T} Y^{T}\right) u+\left(\lambda^{T} X^{T}-\theta X_{0}^{T}\right) v\right\} \\
& \quad= \begin{cases}\theta, & \text { if } Y_{0}^{T}-\lambda^{T} Y^{T} \geq 0, \lambda^{T} X^{T}-\theta X_{0}^{T} \geq 0, \\
-\infty, & \text { if } Y_{0}^{T}-\lambda^{T} Y^{T}<0, \lambda^{T} X^{T}-\theta X_{0}^{T}<0 .\end{cases}
\end{aligned}
$$

Thus, the dual problem is

$$
\begin{array}{ll}
\text { Max } & q(\theta, \lambda) \\
\text { subject to } & \lambda \geq 0,
\end{array}
$$

which reads

$$
\begin{array}{ll}
\operatorname{Max} & \theta \\
\text { subject to } & X \lambda \geq \theta X_{0} \\
& Y \lambda \leq Y_{0} \\
& \lambda \geq 0
\end{array}
$$

Hence, model (34) is the dual of model (33).

Similarly, we can induce the output-oriented models:

$$
\begin{aligned}
& \operatorname{Max}_{u, v} \operatorname{Min}_{j=1, \ldots, n} \frac{\sum_{r=1}^{s} u_{r} y_{r j} / \sum_{i=1}^{m} v_{i} x_{i j}}{\sum_{r=1}^{s} u_{r} y_{r 0} / \sum_{i=1}^{m} v_{i} x_{i 0}} \\
& \text { subject to } \sum_{r=1}^{s} u_{r} y_{r 0}=1 \text {, } \\
& u \geq 0, \quad v \geq 0, \\
& \text { Min } \theta \\
& \text { subject to } \sum_{j=1}^{n} x_{i j} \lambda_{j} \geq x_{i 0} \text {, } \\
& \sum_{j=1}^{n} \lambda_{r j} \lambda_{j} \leq \theta y_{r 0}, \quad \lambda_{j} \geq 0, \\
& j=1, \ldots, n \text {. }
\end{aligned}
$$

\section{Conclusions}

In this paper, we presented a series of two-person games, which further derive various DEA models. Unlike the previous work, the relationship between DEA models and the games is more direct, although the Nash solutions do not generally exist. To sum up, our contribution can be summarized into the following conclusions.

(1) Classical DEA models like the CCR can be viewed as the safest solutions of the game processes, where the $\mathrm{DMU}_{0}$ is one player and the other player is the central evaluator. The $\mathrm{DMU}_{0}$ has the right to choose the 
weights to maximize its performance score, and the evaluator will try to choose a competitive $\mathrm{DMU}_{j}$ to minimize the performance score and has the right to terminate the game.

(2) If the two players exchange their strategy spaces, then we can derive nonclassic DEA models. In these models, $\mathrm{DMU}_{0}$ will choose the worst practice DMU as the reference to compare, instead of the best practice. We can call this kind of models "negative" DEA or "inverted" DEA like Yamada et al. [15] in order to differentiate them from the classical DEA models.

\section{References}

[1] A. Charnes, W. W. Cooper, and E. Rhodes, "Measuring the efficiency of decision making units," European Journal of Operational Research, vol. 2, no. 6, pp. 429-444, 1978.

[2] R. D. Banker, A. Charnes, and W. W. Cooper, "Some models for estimating technical and scale inefficiencies in data envelopment analysis," Management Science, vol. 30, no. 9, pp. 10781092, 1984.

[3] W. Liu, J. Sharp, and Z. Wu, "Preference, production and performance in data envelopment analysis," Annals of Operations Research, vol. 145, no. 1, pp. 105-127, 2006.

[4] J. Zhu, "A buyer-seller game model for selection and negotiation of purchasing bids: extensions and new models," European Journal of Operational Research, vol. 154, no. 1, pp. 150-156, 2004.

[5] R. D. Banker, "A game theoretic approach to measuring efficiency," European Journal of Operational Research, vol. 5, no. 4, pp. 262-266, 1980.

[6] R. D. Banker, A. Charnes, W. W. Cooper, and R. Clarke, "Constrained game formulations and interpretations for data envelopment analysis," European Journal of Operational Research, vol. 40, no. 3, pp. 299-308, 1989.

[7] J. J. Rousseau and J. H. Semple, "Two-person ratio efficiency games," Management Science, vol. 41, no. 3, pp. 435-441, 1995.

[8] J. Semple, "Constrained games for evaluating organizational performance," European Journal of Operational Research, vol. 96, no. 1, pp. 103-112, 1997.

[9] A. Charnes, W. W. Cooper, Z. M. Huang, and D. B. Sun, "Polyhedral Cone-Ratio DEA Models with an illustrative application to large commercial banks," Journal of Econometrics, vol. 46, no. 1-2, pp. 73-91, 1990.

[10] A. Charnes, W. W. Cooper, Z. M. Huang, and D. B. Sun, "Relations between half-space and finitely generated cones in polyhedral cone-ratio DEA models," International Journal of Systems Science, vol. 22, no. 11, pp. 2057-2077, 1991.

[11] G. Hao, Q. Wei, and H. Yan, "Generalized DEA model and the convex cone constrained game," European Journal of Operational Research, vol. 126, no. 3, pp. 515-525, 2000.

[12] C. A. K. Lovell and J. T. Pastor, "Radial DEA models without inputs or without outputs," European Journal of Operational Research, vol. 118, no. 1, pp. 46-51, 1999.

[13] G. E. Halkos and D. S. Salamouris, "Efficiency measurement of the Greek commercial banks with the use of financial ratios: a data development analysis approach," Management Accounting Research, vol. 15, no. 2, pp. 201-224, 2004.
[14] Y. Takamura and K. Tone, "A comparative site evaluation study for relocating Japanese government agencies out of Tokyo," Socio-Economic Planning Sciences, vol. 37, no. 2, pp. 85-102, 2003.

[15] Y. Yamada, T. Matsui, and M. Sugiyama, "An inefficiency measurement method for management systems," Journal of the Operations Research Society of Japan, vol. 37, pp. 158-167, 1994. 


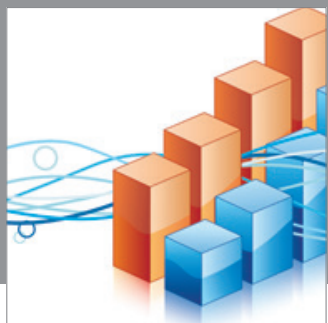

Advances in

Operations Research

mansans

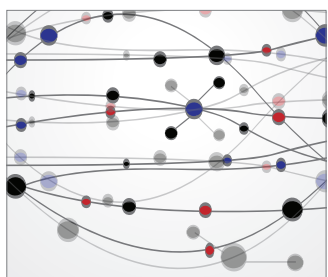

The Scientific World Journal
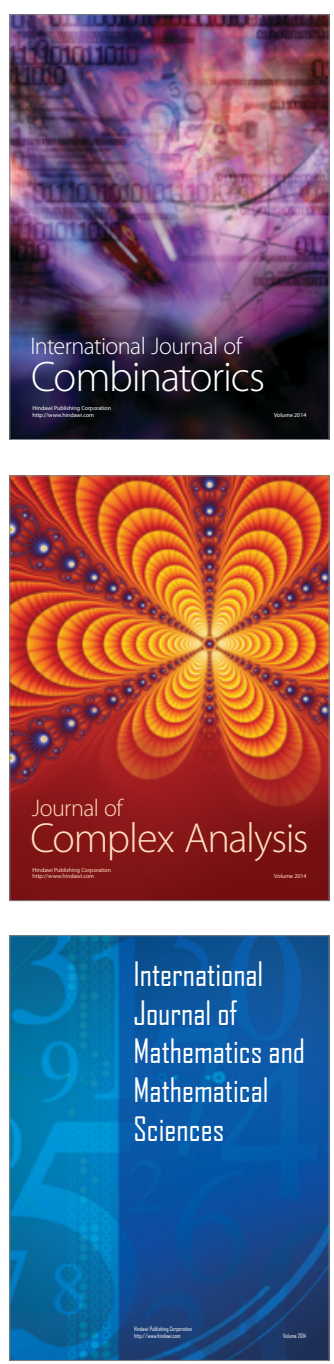
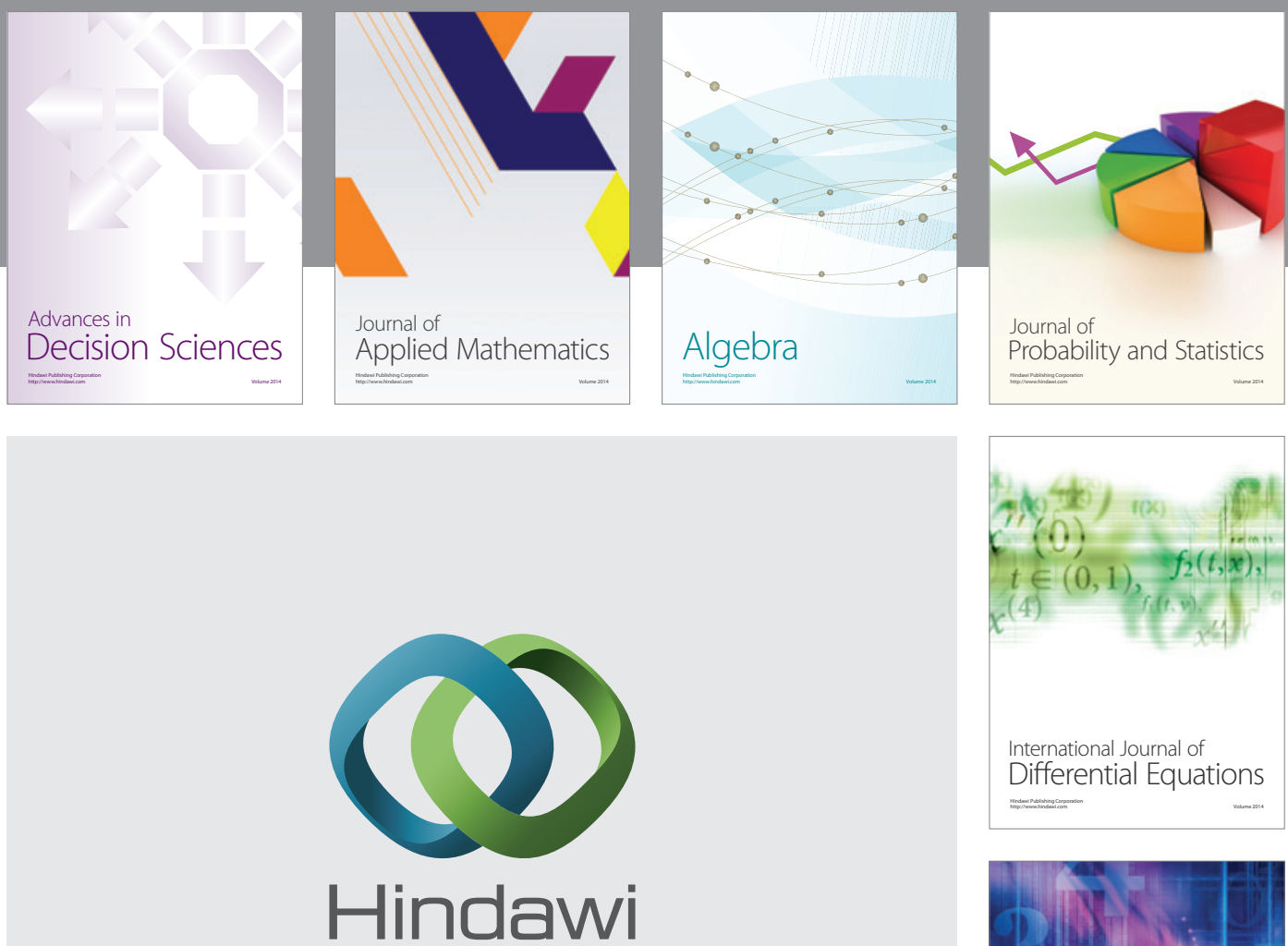

Submit your manuscripts at http://www.hindawi.com
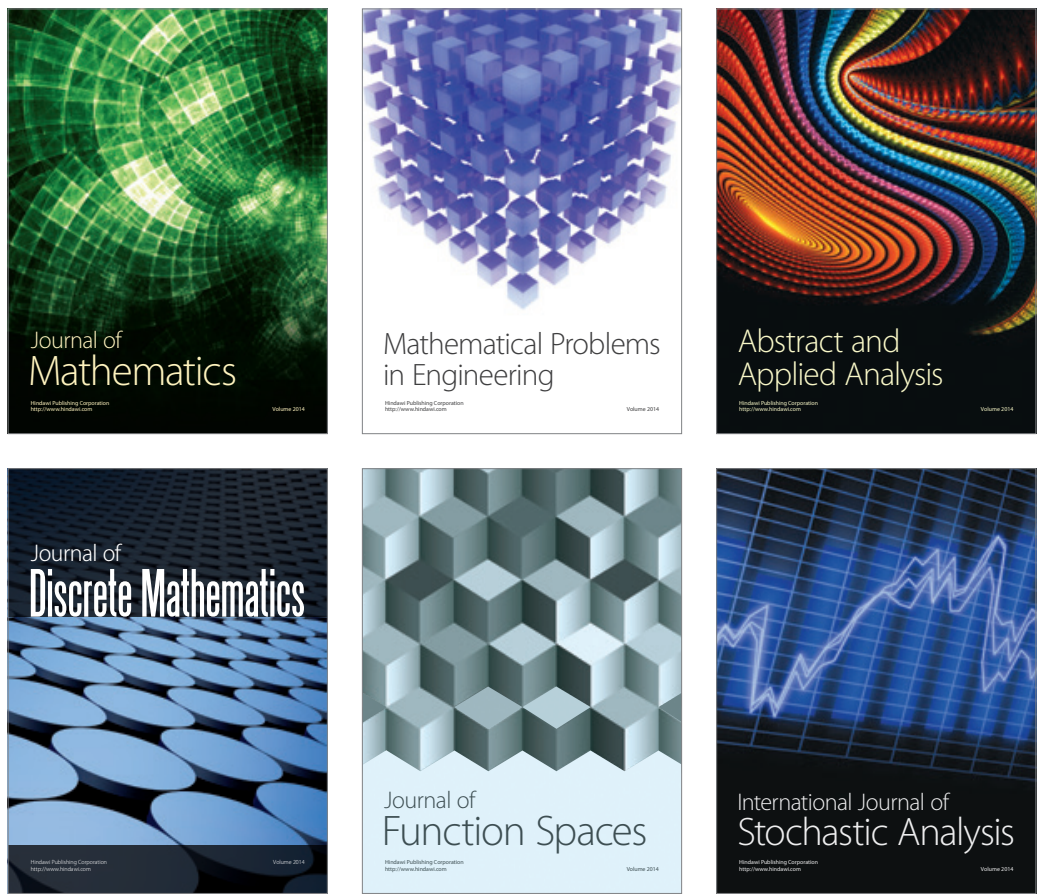

Journal of

Function Spaces

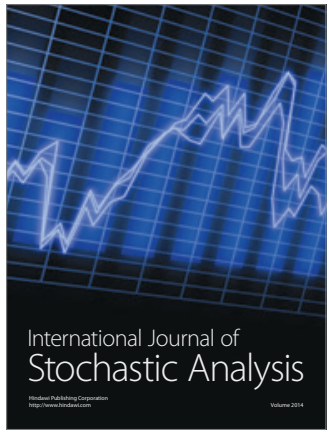

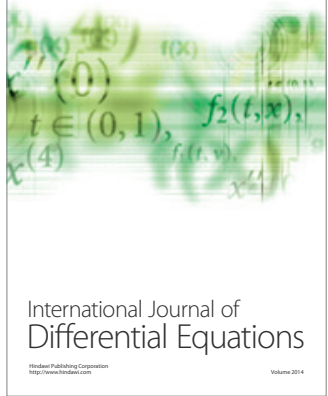
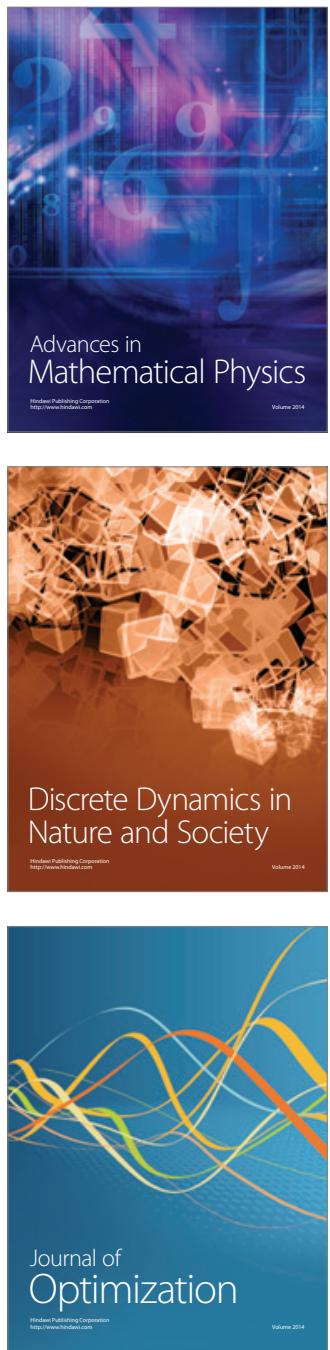